\title{
Advanced characterisation and optical simulation for the design of solar selective coatings based on carbon: transition metal carbide nanocomposites
}

\author{
I. Heras $^{1}$, M. Krause ${ }^{2}$, G. Abrasonis ${ }^{2}$, A. Pardo ${ }^{3}$, J. L. Endrino ${ }^{4}$, E. Guillén ${ }^{1}$, R. Escobar Galindo ${ }^{1^{*}}$ \\ ${ }^{1}$ Abengoa Research S. L., Abengoa, Seville, Campus Palmas Altas 41014, Spain. \\ ${ }^{2}$ Helmholtz-Zentrum Dresden-Rossendorf, Dresden, Germany. \\ ${ }^{3}$ Centre Tecnologic de Manresa, Manresa, Spain \\ ${ }^{4}$ School of Aerospace, Transport and Manufacturing, Cranfield University, MK43z0AL Bedfordshire, UK.
}

\begin{abstract}
Solar selective coatings based on carbon transition metal carbide nanocomposite absorber layers were designed. Pulsed filtered cathodic arc was used for depositing amorphous carbon: metal carbide (a-C:MeC, $\mathrm{Me}=\mathrm{V}, \mathrm{Mo}$ ) thin films. Composition and structure of the samples were characterized by ion beam analysis, X-ray diffraction, Raman spectroscopy, and transmission electron microscopy. The optical properties were determined by ellipsometry and spectrophotometry. Three effective medium approximations (EMA), namely MaxwellGarnett, Bruggeman, and Bergman, were applied to simulate the optical behaviour of the nanocomposite thin films. Excellent agreement was achieved between simulated and measured reflectance spectra in the entire wavelength range by using the Bergman approach, where in-depth knowledge of the nanocomposite thin film microstructure is included. The reflectance is shown to be a function of the metal carbide volume fraction and its degree of percolation, but not dependent on whether the nanocomposite microstructure is homogeneous or a self-organized multilayer. Solar selective coatings based on an optimized a-C:MeC absorber layer were designed exhibiting a maximum solar absorptance of $96 \%$ and a low thermal emittance of $\sim 5$ and $15 \%$ at 25 and $600^{\circ} \mathrm{C}$, respectively. The results of this study can be considered as a predictive design tool for nanomaterial-based optical coatings in general.
\end{abstract}

\section{Introduction}

Concentrating solar power (CSP) is a commercially available technology in the field of renewable energies. It is based on the conversion of concentrated solar energy into thermal and subsequently electrical energy. Nowadays, the two most prominent commercial CSP technologies are parabolic trough collectors and central receiver tower plants. Main components of CSP technology are solar light concentrators (reflectors), solar receivers

\footnotetext{
* Corresponding author. Tel +34 649833 073; e-mail address: ramon.escobar@abengoa.com (R. EscobarGalindo)
} 
(absorbers), heat transport and storage systems and electrical power generators [1]. Solar selective coatings with high absorptance $(\alpha)$ in the solar spectral range $(0.3-2.5 \mu \mathrm{m})$ and low thermal emittance $\left(\varepsilon_{T}\right)$ in the infrared region $(2.5-30 \mu \mathrm{m})$ are required for solar receivers [2]. Physical vapor deposited (PVD) solar selective coatings have been widely employed in parabolic trough systems, where temperatures below $400{ }^{\circ} \mathrm{C}$ and vacuum environments are applied [3]. However, these solutions cannot be directly implemented in central receiver tower plants that operate at higher temperatures in air. Nowadays, commercial paints such as Pyromark ${ }^{\circledR}$ are the most commonly employed absorber coatings in tower plant central receivers. Their disadvantages are a poor solar selectivity ( $\varepsilon=86 \%$ at room temperature) and a fast degradation during operation. The latter one requires permanent maintenance and repairs typically every two years [4]. In a recent review [3], an extensive variety of PVD coatings was evaluated. Correspondingly, the coatings degrade easily at high temperatures $\left(>400^{\circ} \mathrm{C}\right)$ in air due to oxidation and/or diffusion of the metal substrate into the coatings. These examples underline the necessity to develop novel types of solar selective coatings that are characterized by high-temperature stability in oxidizing environments.

State of the art designs of solar selective coatings include multilayers comprised of an antireflective layer (AR), an absorber layer, and an infrared reflection (IR) layer. Antireflective layers such as a $\mathrm{Si}_{3} \mathrm{~N}_{4}$ or $\mathrm{Al}_{2} \mathrm{O}_{3}$ located on the top of the coating improve the solar absorption and stabilize the coating at high temperatures [5]. The infrared reflective layer placed under the absorber layer reduces the thermal emittance at high temperatures and acts as a thermal barrier to avoid material diffusion between the absorber material and the substrate [6].

Solar absorber layers based on nanocomposite materials were investigated in numerous studies [7,8]. Among them, a-C:MeC nanocomposites are suitable candidates for hightemperature solar absorber applications [9]. Pure a-C thin films are good candidates for solar absorber materials [10] but they are not air stable at temperatures above $300^{\circ} \mathrm{C}$. In combination with group 4, 5, and 6 transition metals, carbon forms a number of exceptionally stable interstitial carbides. These carbides are characterized by high melting points (in the range of $2600-2800^{\circ} \mathrm{C}$ [11]), high thermal and electrical conductivity, and high reflectivity in the entire UV-Vis-IR spectral range [12]. The presence of these carbides stabilizes the nanocomposite microstructure at high temperatures $[13,14]$. Different microstructures of the a-C:MeC nanocomposites have been reported i) carbide phases dispersed in the carbon matrix in the form of nanoparticles, with different morphologies (globular or elongated $[15,16]$ ) and crystal structures (amorphous [17] or crystalline [18]) and ii) a self-forming multilayer 
structure with periodic concentration fluctuations of metal in a-C [19]. These systems can exhibit improved mechanical properties (lower intrinsic compressive stress, improved adherence to the substrate or better wear resistance [20]) compared to pure a-C films.

Combining the advantageous optical and thermo-mechanical properties of the individual components can result in adjustable solar selective properties for a-C:MeC nanocomposites with high-temperature resistance. Harding et al. reported that sputter deposited metal carbides $(\mathrm{Cr}, \mathrm{Fe}, \mathrm{Mo}, \mathrm{Ni}$, Ta and $\mathrm{W})$ on copper substrates show good selective properties $(\alpha>80 \%$ and $\varepsilon_{600^{\circ} \mathrm{C}} \sim 7 \%$ ) [21,22]. Gampp developed a multilayer coating based on hydrogenated a-C codeposited with $\mathrm{Cr}$ or $\mathrm{W}$ by sputtering (a-C:H/Cr and a-C:H/W [23-25]). Yin et al. [26] deposited a-C:H/SS-a-C:H (SS: stainless steel) using cathodic arc technique. However, in all these examples the optical properties dropped drastically for temperatures higher than $600^{\circ} \mathrm{C}$ due to different types of degradation mechanisms: i) undesired interaction between coatings and the metallic bottom layer and ii) degradation due to the intrinsic instability of the coating material itself. More recently, Schüler et al. developed a multilayer coating based on titanium and amorphous hydrogenated carbon (a-C:H/Ti) films [27] adding silicon to increase the durability in air.

The proper determination of the optical constants of the nanocomposites represents a major difficulty for the optimization of a-C:MeC solar absorber coatings up to now. This is particularly caused by the limited knowledge on the dependences of optical properties in the solar range on nanocomposite film composition, microstructure and phase morphology. In heterogeneous materials with nanoparticles much smaller than the light wavelength (such as a-C:MeC thin films), the optical properties can be described by so-called effective dielectric functions or effective medium approximations (EMA) [28]. They give a non-trivial average of the dielectric functions of the individual components of the nanocomposite in the form of an effective dielectric function $\left(\varepsilon_{e f f}\right)$ for the composite material.

Several EMAs have been established, Maxwell-Garnett (MG) [29] and Bruggeman (BRU) being the most widely used ones [30]. In both, the final effective dielectric function depends on the volume fraction of the particle material and on the optical constants of the individual constituents. Characteristic properties of the microstructure or the shape of the embedded particles are not incorporated. In contrast, Bergman (BER) representation [31] applies several corrections related to the shape of the nanoparticles and the degree of percolation of the embedded nanoparticles, which improve the accuracy of the simulated effective dielectric function. This approximation is especially useful in cases where the microstructure plays a significant role in the optical properties of the heterogeneous material. 
By now the design of absorber coatings relies very often on an empirical ad-hoc approach. A smart coating development, however, would require a reliable simulation of the optimized coating stack as the first and the experimental confirmation as the second step. The former would have to identify and incorporate those structure elements that are relevant for the optical response. Therefore, the first objective of this study is to apply such approach to model the optical properties of a-C:VC and a-C:MoC nanocomposite films as a solar absorber layer. The second objective is to design a complete solar selective AR layer / a-C:MeC absorber layer / IR reflector stack by using this model and to find out the theoretical limit of the absorbance at RT and at temperatures relevant for high-temperature absorber applications.

In this study, a-C:VC and a-C:MoC nanocomposite thin films with different metal carbide contents and different microstructures have been deposited by pulsed filtered cathodic vacuum arc (PFCVA). Among the different physical vapour deposition (PVD) techniques, FCVA has the highest plasma ionization ratio. This results in denser thin films with higher hardness and better adhesion [32], and allows the control of the composition ratio of absorbing carbon and reflecting/refractory carbide. The element composition of the nanocomposites was determined using Rutherford Backscattering Spectrometry (RBS) and Nuclear Reaction Analysis (NRA). Their structure was characterized by Raman spectroscopy, $\mathrm{X}$-ray diffraction (XRD), and high-resolution transmission electron microscopy (HRTEM). Reflectance spectra were obtained by UV-Vis-NIR spectrophotometry, and the optical constants were measured by spectroscopic ellipsometry (SE). The optical properties were simulated comparing three different EMAs, namely Maxwell Garnett, Bruggeman and Bergman using the commercial software CODE [33]. The Bergman EMA was found to be the most suitable one for this type of materials. The dielectric functions obtained by the simulation were further used for a detailed examination of the absorptance of a-C:MeC $(\mathrm{Me}=$ $\mathrm{V}, \mathrm{Mo}$ ) layers. Based on this approach an optimized, complete stack with an outstanding solar absorptance $(\alpha)$ of $96 \%$ and an emittance $\left(\varepsilon_{T}\right)$ of $5 / 15 \%$ at $25 / 600^{\circ} \mathrm{C}$ was predicted. This is significantly better than the values reported for this class of materials so far, which were an $\alpha=91.0 \%$ with $\varepsilon_{100^{\circ} \mathrm{C}}=11 \%$ for an a-C: $\mathrm{TiC} / \mathrm{SiO}_{2}$ stack on $\mathrm{Cu}$ substrate [27,34].

\section{Experimental details}

\subsection{Thin film growth}

Thin films were simultaneously grown on single crystalline Si (100) and on polished Inconel ${ }^{\circledR} 625$ substrates in the same deposition batches. The former ones were used for Rutherford Backscattering Spectroscopy (RBS), Raman and XRD characterization while the 
latter were employed for high-resolution transmission electron microscopy (HRTEM) and optical characterization. Before deposition, the substrates were cleaned in an ultrasonic bath in distilled water, acetone and ethanol.

The depositions were carried out using a PFCVA system equipped with two arc sources, PFCVA-450 from Plasma Technology Limited [35] (Figure 1). The system includes electromagnetic filters in the curved ducts to reduce the incorporation of macroparticles in the growing films. The generated arc plasmas are guided into the vacuum chamber by an electromagnetic field applied to the curved ducts. External solenoid coils are wrapped around the ducts and produce an axial magnetic field. The coils are biased with $20 \mathrm{~V}$ and a current of 15 A to build up a lateral electrical field.

The substrates were situated on a rotating holder in the center of the chamber, $240 \mathrm{~mm}$ away from the exit of the duct. A negative pulse bias voltage of $-400 \mathrm{~V}(100 \mathrm{kHz}, 20 \%$ duty cycle $)$ was applied to the substrates during film deposition in order to improve adhesion and to release the residual stress of the coating [36]. The sample holder was rotated with a continuous rotation speed of $1.4 \mathrm{rpm}$. The deposition chamber was evacuated to a pressure of $2 \cdot 10^{-3} \mathrm{~Pa}$ before deposition. During film deposition, an argon flow of $3.5 \mathrm{sccm}$ was introduced into the chamber to stabilize the arc, and the working pressure was kept constant at $0.11 \mathrm{~Pa}$ by a pressure control valve.

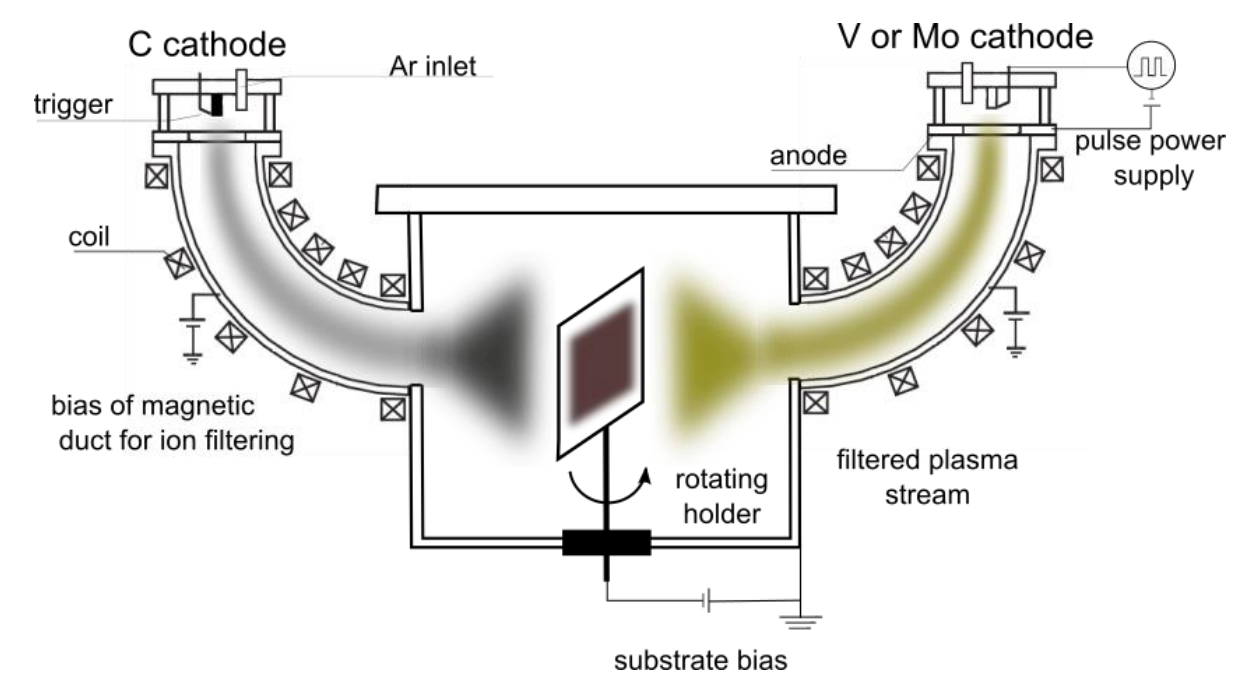

Figure 1: Schematic diagram of the pulsed filtered cathodic vacuum arc (PFCVA) system equipped with two cathodic arc sources.

One arc source is used as carbon cathode and the other as a metallic one (vanadium or molybdenum). a-C:MeC coatings were prepared by simultaneous co-evaporation of both cathodic arc sources. The discharge pulse duration was $3 \mathrm{~ms}$ at a repetition rate of $7.5 \mathrm{~Hz}$, with adjustable intensity. The deposition time was 30 minutes. All the deposition parameters are summarized in Table 1. 
Table 1: Deposition parameters of the $\mathrm{C}$ and a-C:MeC thin films deposited with PFCVA. All the samples were deposited at room temperature on $\mathrm{Si}(100)$ and Inconel substrates

\begin{tabular}{ll}
\hline Parameter & Value \\
\hline Base pressure (Pa) & $2.00 \cdot 10^{-3}$ \\
Working pressure (Pa) & 0.11 \\
Argon flow (sccm) & 3.5 \\
Deposition time for a-C:MeC samples (min) & 30 \\
Deposition time for C (min) & 60 \\
Duct bias (V) & -20 \\
Duct current (A) & 15 \\
Axial magnetic field (G) & 200 \\
Cathodes & $99.997 \% \mathrm{C}$ \\
& $99.993 \% \mathrm{~V}$ \\
& $99.995 \% \mathrm{Mo}$ \\
Cathode diameter (mm) & 10 \\
Discharge current for carbon (A) & 2.5 \\
Discharge current for high/ low metal content (A) & $5 / 1.25$ \\
Pulse duration [frequency] of the discharge (ms) [Hz] & $3[(7.5]$ \\
Bias voltage at deposition stage (V) & $-400(100 \mathrm{kHz}$ pulsed) \\
Duty cycle of the bias (\%) & 20 \\
Rotation speed of the substrate (rpm) & 1.4 \\
Filter to the substrate distance (mm) & 240 \\
\hline
\end{tabular}

Both carbon - vanadium carbide (a-C:VC) and carbon - molybdenum carbide (a-C:MoC) thin films were deposited with two different metal concentrations, denoted as high (h) and low (l) throughout this article. The metal concentration was controlled via the cathode current ratio $R$, given by $R=I_{\text {carbon }} / I_{\text {metal }}$. Therefore, the carbon arc source current was kept constant and the metal arc source current was varied in such a way that $R$ was either 0.5 for high metal content samples and 2.0 for low metal content ones.

A pure carbon reference film was prepared by applying the same deposition conditions expect of using only the carbon arc source and extending the deposition time to 60 minutes.

\subsection{Thin films microstructure characterization}

The carbon and metal (V or Mo) areal densities of films grown on Si substrates were determined by a combination of Rutherford Backscattering Spectroscopy (RBS) and Nuclear Reaction Analysis (NRA) measurements. The RBS intensity of scattered deuterium ions is proportional to the atomic number $\mathrm{Z}$ of all elements of the sample. Here it was used for the determination of the metal contents. Since the RBS cross section of carbon is low and the carbon signal is superimposed to that of the Si substrate, NRA measurements were employed to accurately determine carbon areal densities.

The RBS and NRA measurements were carried out at a $2 \mathrm{MeV}$ Van der Graff and a $3 \mathrm{MeV}$ Tandem accelerator, respectively. The RBS spectra were obtained by using ${ }^{4} \mathrm{He}$ ions with energy of $1.7 \mathrm{MeV}$. The data were acquired with a silicon barrier detector located at a backscattering angle of $170^{\circ}$, whose energy resolution was $13 \mathrm{keV}$. The simulation software 
SIMNRA [30] version 6.06 was used to extract the metal areal density. NRA was measured by using 1.248 MeV deuterium ions as incident particles at a detection angle of $135^{\circ}$. The nuclear reaction ${ }^{12} C(d, p){ }^{13} C$ has its maximum cross section at an incident deuterium ion energy of around $1.248 \mathrm{MeV}$. The intensity of the emitted proton radiation is proportional to the carbon concentration in the nanocomposite thin film [37]. The carbon content was determined quantitatively by integrating the NRA intensity and comparing it with that of a C:Ni reference sample.

The thickness and roughness of the deposited samples were measured using a 50x objective Confocal Microscope (Pl $\mu$ 2300, SENSOFAR).

The phase structure of the carbon matrix was determined by Raman spectroscopy. MicroRaman spectra were recorded on a LabramHR spectrometer (Horiba, Germany), which is equipped with a liquid $\mathrm{N}_{2}$ cooled charge-coupled device detector and coupled to a $\mathrm{BH} 2$ microscope (Olympus, Germany). The laser beam of a Nd:YAG solid-state laser with a wavelength of $532 \mathrm{~nm}$ was focussed to a spot diameter of $1 \mu \mathrm{m}$ using a 50-fold magnifying objective. The laser power at the sample was $1 \mathrm{~mW}$. The scattered light was collected in an $180^{\circ}$ backscattering geometry and dispersed by a 300 line $/ \mathrm{mm}$ grating. No sample degradation occurred under these conditions. For the analysis of the Raman line shape, the software PeakFit (version 4.12, Seasolve Software Inc.) was employed. The measured spectra were fitted with a combination of a linear background fixed at the noise level of the spectra at 850 $2000 \mathrm{~cm}^{-1}$, a symmetric Lorentzian shape for the $D$-peak, and an asymmetric Breit-WignerFano (BWF) line for the $G$-peak $[13,38,39]$. The intensity $I$ as a function of the wavenumber $\omega$ for the BWF line shape is described by equation (1):

$$
I(\omega)=\frac{I_{0}\left[1+2\left(\omega-\omega_{0}\right) / q \Gamma\right]^{2}}{1+\left[2\left(\omega-\omega_{0}\right) / \Gamma\right]^{2}}
$$

with the intensity maximum $\left(I_{\max }\right)$

$$
I_{\max }=I_{0}\left[1+1 / q^{2}\right]
$$

positioned at

$$
\omega_{\max }=\omega_{0}+\Gamma / 2 q,
$$

where $I_{0}$, is the peak intensity, $\omega_{0}$ the peak position, $\Gamma$ the FWHM, and $q$ is the BWF coupling coefficient. The intensity maximum defined by equation (2) will be used to denote the intensity of the $G$ and $D$ peaks and equation (3) to define their wave number.

The phase structure of the dispersed phase in the a-C:MeC thin films was determined by $\mathrm{X}$ ray diffraction employing grazing incidence geometry (GIXD) using a PANalytical X'Pert PRO diffractometer with $\mathrm{Cu}-K_{\alpha}$ radiation $\left(\lambda=1.5406 \AA\right.$ ). The incident angle was $0.4^{\mathrm{o}}$, and the 
XRD patterns were measured in the diffraction angle range of $30-100^{\circ}$ in steps of $0.05^{\circ}$. The crystallite size of the main crystal directions was estimated employing the Scherrer equation with $k$ of 0.94 [40].

The microstructure was analysed by cross-sectional HRTEM with a TECNAI F30 microscope operated at $300 \mathrm{kV}$ accelerating voltage. It is equipped with Schottky-type field emission gun and an ultra-high resolution pole piece. The thin lamellas required for the cross-section analysis were made using a Focused Ion Beam (FIB) Dual Beam Helios 650, consisting of a $30 \mathrm{kV}$ Ga focused ion beam combined with a $30 \mathrm{kV}$ electron beam placed at $52^{\circ}$ between them, and with the Omniprobe ${ }^{\circledR}$ manipulator. Sample-protecting Pt thin films were deposited by electron beam deposition of $\left(\mathrm{CH}_{3}\right)_{3}\left(\mathrm{C}_{p} \mathrm{CH}_{3}\right) \mathrm{Pt}$.

\subsection{Determination of optical properties}

The specular reflectance at room temperature was measured in the range 250 to $3300 \mathrm{~nm}$ using a Shimadzu UV-Vis-NIR Solid Spec-3700 spectrophotometer under an incident angle of $5^{\circ}$ from the normal. The system is equipped with three detectors (PMT, PbS and InGaAs) which ensure sufficient sensitivity in the whole wavelength range. An aluminum mirror was used as a reference and the reflectance was corrected by using a calibrated Al standard. The software CODE was employed to simulate the reflectance spectra.

Based on the reflectance spectrum, the solar absorptance $(\alpha)$, defined as the fraction of incident radiation in the solar wavelength range that is absorbed, is calculated according to equation (4) [41]:

$$
\alpha=\frac{\int_{\lambda_{1}}^{\lambda_{2}}[1-R(\lambda)] G(\lambda) d \lambda}{\int_{\lambda_{1}}^{\lambda_{2}} G(\lambda) d \lambda}
$$

where $\lambda_{1}=300 \mathrm{~nm}$ and $\lambda_{2}=2500 \mathrm{~nm}$ denote the integration limits, $R(\lambda)$ the spectral reflectance, and $G(\lambda)$ is the reference solar spectrum AM 1.5. The thermal emittance is calculated applying Kirchoff's law from the reflectance spectra in the infrared region, following equation (5):

$$
\varepsilon(T)=\frac{\int_{\lambda_{1}}^{\lambda_{2}}[1-R(\lambda, T)] B(\lambda, T) d \lambda}{\int_{\lambda_{1}}^{\lambda_{2}} B(\lambda, T) d \lambda},
$$

where $B(\lambda, T)$ is the spectral distribution of the blackbody radiation calculated with Plank's law [42] for a specific temperature $T$ in Kelvin degrees. The integration interval is limited by $\lambda_{1}=1 \mu \mathrm{m}$ and $\lambda_{2}=25 \mu \mathrm{m}$, based on European standard EN-673:2011 [43]. 


\section{Results and discussion}

\subsection{Film composition, density and roughness}

In order to calibrate the equivalent areal density for each metal content, data obtained in RBS experiments of samples with the highest vanadium and molybdenum content (a-C:CV (h) and a-C:MoC (h) samples) were used. The RBS spectra (Figure 2) show the V and Mo metal signals at approximately 1280 and $1480 \mathrm{keV}$, respectively. Due to the high $\mathrm{Z}$ of both metals, these signals are strong and clearly differentiated from the overlapping signals of the silicon substrate and carbon below $900 \mathrm{keV}$ and at $440 \mathrm{keV}$, respectively. The weak peak around $1100 \mathrm{keV}$ is caused by the incorporation of less than 3 at.\% Ar used to stabilize the arc formation during the deposition. A closer look at the vanadium RBS signal in a-C:VC (h) (inset in Figure 2a) reveals an inhomogeneous depth distribution of the metal. In fact, in order to fit the spectrum properly, five different layers with alternating high (22 at.\%) and low (17 at.\%) vanadium content were taken into account. To a lesser extent, this depth inhomogeneity of the metal concentration is also observed in the a-C:MoC (h) nanocomposite. In this case, it was necessary to assume three different regions with 17, 25, and 14 at.\% Mo to fit the RBS spectrum properly. These results will be discussed in detail later in combination with HRTEM images.
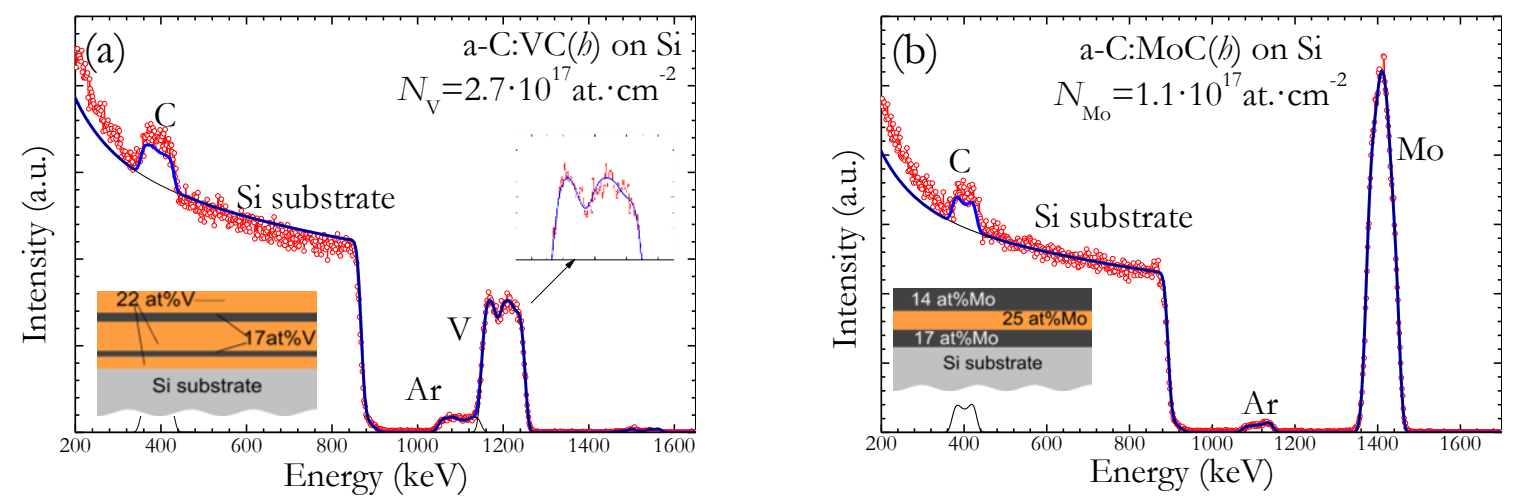

Figure 2: RBS spectra of (a) a-C:VC and (b) a-C:MoC thin films with high metal content, to calculate the equivalent metal concentration. The modulation of the composition in the a-C:VC samples is represented in the inset of (a)).

The nuclear reaction of the carbon atoms with deuterium ions gives rise to the proton signal at approximately $3100 \mathrm{keV}$ [54], while the backscattered deuterium ions contribute to the RBS part of the spectrum (Figure 3). At the measurement conditions, 1000 counts correspond to $5.77 \cdot 10^{17}$ carbon atoms $/ \cdot \mathrm{cm}^{2}$, as determined from a reference C:Ni sample, and $2.7 \cdot 10^{17}$ and $1.1 \cdot 10^{17}$ atoms $\mathrm{cm}^{-2}$ for vanadium and molybdenum, respectively, extracted from RBS analysis. The total amount of carbon and metal atoms deposited per unit area $\left(t_{C}\right.$ and $t_{M e}$ respectively) was determined by integrating the peak areas of the NRA and RBS signals for 
each sample and subsequent summation. The metal-carbon ratio in percent was calculated as shown in equation (6):

$$
\text { at. } \%(M e)=\frac{t_{M e}}{t_{M e}+t_{C}} x 100 \% \text {. }
$$
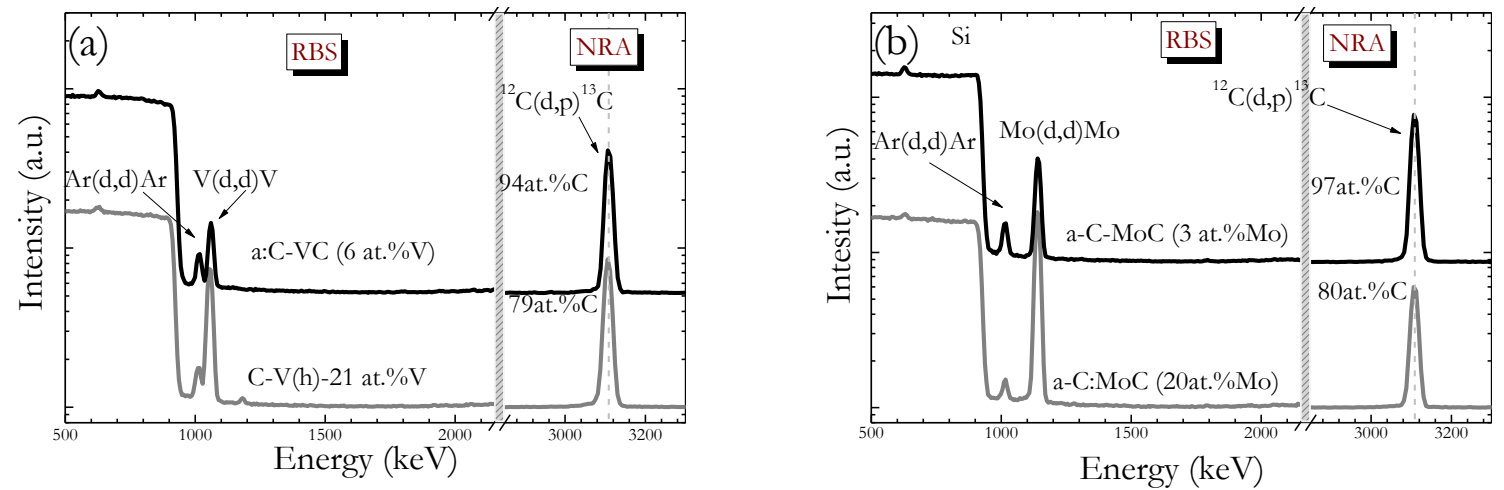

Figure 3: NRA spectra of (a) a-C:VC and (b) a-C:MoC for high and low metal content films. Two distinct regions in the spectra are indicated as RBS and NRA, corresponding to backscattered deuterium signal and nuclear reaction proton signal, respectively.

The averaged metal contents of the samples are given in Table 2 . The film density can be estimated from the RBS areal density and film thickness [44]. In the case of a-C:VC (h) sample, the density is $\sim 3.0 \mathrm{~g} \cdot \mathrm{cm}^{-3}$, i.e. lower than the density of bulk vanadium carbide $(5.7$ $\left.\mathrm{g} \cdot \mathrm{cm}^{-3}[45]\right)$ and higher than in amorphous carbon $\left(2.1 \cdot \mathrm{cm}^{-3}[46]\right)$ typically obtained with cathodic arc deposition. In the case of the a-C:MoC (h) sample, the estimated average density is $4.8 \mathrm{~g} \mathrm{~cm}^{-3}$.

The thickness, deposition rate and roughness of the samples grown on silicon are summarized in Table 2. The deposition rate of samples deposited on Inconel was by a factor of $1.39 \pm 0.03$ $\mathrm{nm} / \mathrm{min}$ higher than those on Si. It is worth noting that the average roughness $\left(R_{a}\right)$ is below 3 $\mathrm{nm}$ for the films grown on Si substrates. This indicates the formation of ultra-smooth surfaces due to the effective removal of the macroparticles with the curved filters.

Table 2: Metal content, thickness, deposition rate and roughness of PFCVA deposited thin films on silicon substrates.

\begin{tabular}{lcccc}
\hline Sample & $\begin{array}{c}\text { Metal content } \\
(\text { at. } \%)\end{array}$ & $\begin{array}{c}\text { Thickness } \\
(\mathrm{nm})\end{array}$ & $\begin{array}{c}\text { Deposition rate } \\
(\mathrm{nm} / \mathrm{min})\end{array}$ & $R_{a}(\mathrm{~nm})$ \\
\hline a-C:VC (high) & $21 \pm 1$ & $140 \pm 5$ & $4.8 \pm 0.2$ & $2.5 \pm 0.2$ \\
a-C:VC(low) & $6 \pm 1$ & $110 \pm 10$ & $3.7 \pm 0.2$ & $2.7 \pm 0.2$ \\
a-C:MoC (high) & $19 \pm 1$ & $90 \pm 15$ & $3.0 \pm 0.2$ & $2.7 \pm 0.2$ \\
a-C:MoC (low) & $3 \pm 1$ & $125 \pm 10$ & $3.3 \pm 0.2$ & $2.8 \pm 0.2$ \\
a-C & 0 & $150 \pm 5$ & $2.5 \pm 0.2$ & $3.1 \pm 0.2$ \\
\hline
\end{tabular}

\subsection{Carbon bonding as revealed by Raman spectroscopy}

The Raman spectrum of the pure carbon film exhibits one peak that is asymmetrically broadened towards smaller Raman shifts (Figure 4). Line shape analysis reveals its complex structure, composed of a shoulder and a main peak in the range of the characteristic $s p^{2}$ carbon $\left(s p^{2}-\mathrm{C}\right) D$ line and $G$ line. Based on the low $I_{D} / I_{G}$ ratio of 0.40 and the $G$ line position 
of $1544 \mathrm{~cm}^{-1}$, the phase structure of the carbon reference corresponds to a-C with a low degree of six-fold $s p^{2}-\mathrm{C}$ ring clustering.
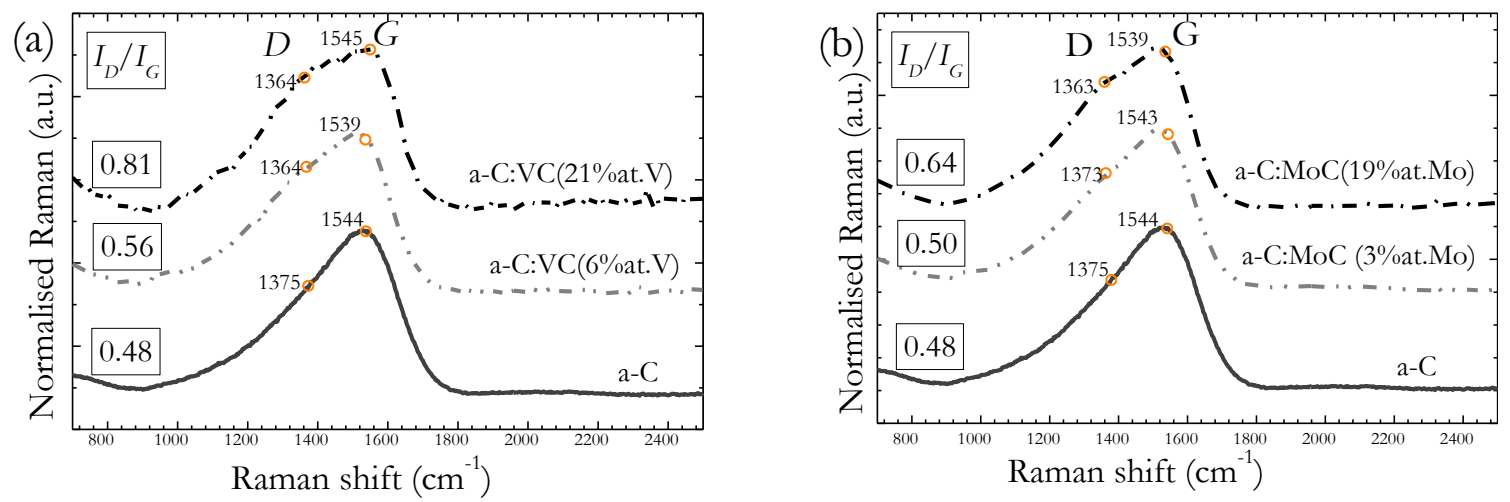

Figure 4: (a) Normalized Raman spectra of a-C:VC deposited films with 21 and 6 at.\% V and (b) a-C:MoC films with 19 and 3at.\%Mo content. The Raman spectrum of the deposited carbon sample is represented as a reference.

The Raman spectra of the a-C:MeC nanocomposite films exhibit a shape that is constituted of a shoulder in the range of the $D$-line and a maximum at the Raman shift of the G line of $s p^{2}$ C. The ratio of the peak intensities $I_{D} / I_{G}$ increases with increasing metal content from 0.50 for a-C:MoC (3 at.\% Mo) via $0.56(6$ at. $\% \mathrm{~V})$ and 0.64 (19 at. \% Mo) to 0.80 for a-C:VC (21 at.\% V). The position of the $\mathrm{G}$ line is observed at 1540 to $1545 \mathrm{~cm}^{-1}$ for all samples. The three-stage model of Ferrari and Robertson [47] implies a $s p^{2}-\mathrm{C}$ phase consisting of a-C with a higher degree of 6-fold aromatic ring clusters than in the reference sample of pure carbon. The corresponding $s p^{3}-\mathrm{C}$ content of the carbon matrix in all the a-C:MeC samples is of the order of 10 to $15 \%$. The increasing $I_{D} / I_{G}$ ratio reveals that the metal addition promotes formation of 6-fold aromatic rings in the amorphous carbon matrix of both a-C:MeC types [48] in comparison to pure carbon films.

\subsection{Phase structure of the nanocomposites}

$\mathrm{X}$-ray diffractograms of the a-C:MeC samples deposited on Si substrate are shown in Figure 5. The diffraction pattern of the a-C:VC film (21 at.\% V) shows four narrow Bragg peaks positioned at $2 \theta=38.1^{\circ}, 44.3^{\circ}, 64.4^{\circ}$ and $77.5^{\circ}$ (Figure 5a). These peaks are assigned to interferences of the (111), (200), (220) and (222) planes of $f c c$ vanadium carbide [49,50], respectively. Applying Bragg's equation the lattice constant (4.13 $\AA$ ) is obtained in good agreement with the reference value (4.16 $\AA$ ). Employing Scherrer equation with 0.94 as the value of the form factor $K$, a minimum crystal size of (27 \pm 10$) \mathrm{nm}$ was estimated. The second set of interferences is observed at $2 \theta$ values of $36.7^{\circ}, 42.7^{\circ}, 63.0^{\circ}$, and $74.9^{\circ}$. The diffraction angles are slightly smaller than those of the first set, and moreover, the peaks are significantly broader. The diffractogram pattern points to a second $f c c$-VC structure with a lattice constant of $4.25 \AA$ and a minimum crystal size of $(7 \pm 4) \mathrm{nm}$. The coexistence of two $f c c$-VC phases 
can be rationalized by assuming regions with a different $\mathrm{V}$ content, what is consistent with the observed fine structure of the RBS signal. The data are in qualitative agreement with those of a recent study on magnetron sputtered a-C:CV films [39]. Similar as in the present study, a slight decrease of the VC lattice constant with increasing V content was reported therein. Moreover, the minimum crystal size increased from $(1.7 \pm 0.2) \mathrm{nm}$ for 19 at. $\% \mathrm{~V}$ to $(6 \pm 1)$ $\mathrm{nm}$ for 27.9 at.\% V. No significant diffraction peaks were identified in the low metal content a-C:VC sample with 6 at. \% V (Figure 5 a).
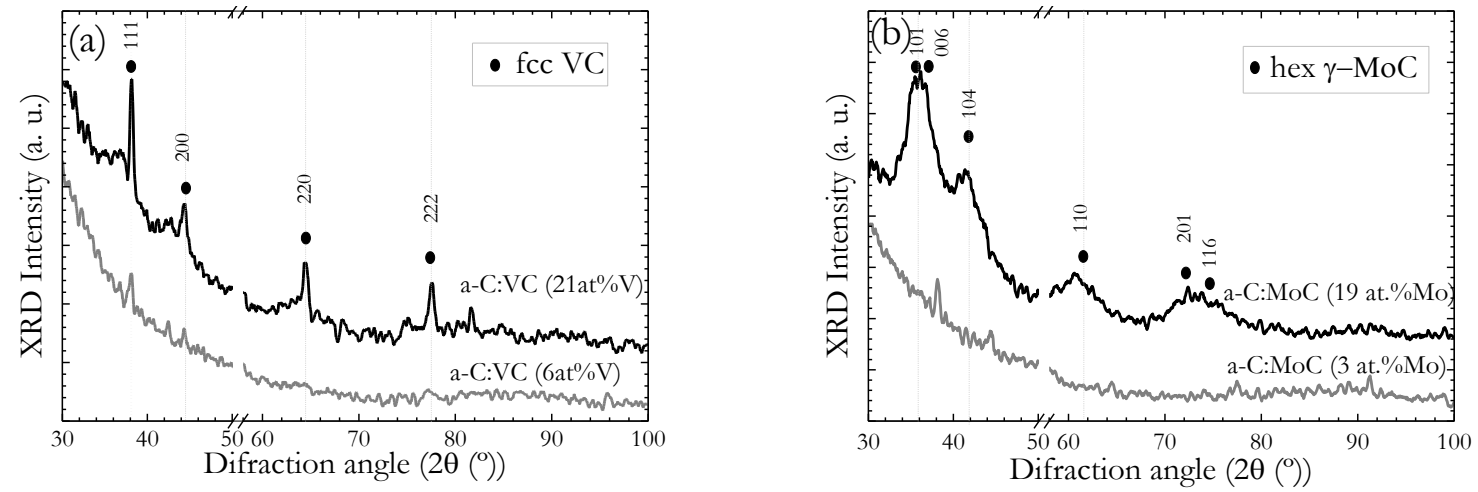

\footnotetext{
Figure 5: XRD patterns of (a) a-C:VC and (b) a-C:MoC for samples with high and low metal content. The dotted lines indicate the more significant peaks position of the referred crystal phases. The patterns are intercepted between $\sim 50$ and $58^{\circ}$ in order to omit the intense $\mathrm{Si}$ (311) peak at $\sim 52^{\circ}$ of the $\mathrm{Si}(100)$ substrate
}

Figure $5 \mathrm{~b}$ shows the XRD pattern of the a-C:MoC samples. The diffractogram of a-C:MoC $\left(\sim 19\right.$ at.\% Mo) shows four broad diffraction peaks at $2 \theta=36^{\circ}, 41^{\circ}, 61^{\circ}$ and $73^{\circ}$. The pattern closely resembles that of simple hexagonal monocarbide $\gamma$-MoC [49]. Its main peaks are tabulated at $2 \theta=\sim 36^{\circ}, 42^{\circ}, 61^{\circ}$ and $73^{\circ}$ and assigned to the crystal planes (101), (104), (110) and (116), respectively. The main peaks observed here for a-C:MoC ( $\sim 19$ at.\% Mo) are also close to those of $h c p \beta-\mathrm{Mo}_{2} \mathrm{C}$ [49]. According to the binary phase diagram of Mo and C [51], the thermodynamically stable phase for a Mo content of $\leq 50$ at. $\%$ is $\gamma$-MoC with hexagonal simple space group P6m2. Therefore we tentatively assign the observed diffractogram of aC:MoC (19 at.\% Mo) to the $\gamma$-MoC phase. The phase structure of a-C:MoC (19 at.\% Mo) will be further discussed in the subsequent section about the HRTEM analysis. Scherrer equation gives a minimum $\gamma$-MoC crystallite size of $3.5 \pm 0.5 \mathrm{~nm}$.

For a-C:MoC (3 at.\% Mo), no significant diffraction peaks were identified. As for a-C:VC (6 at.\% V), the low metal content is presumably responsible for a small number of $\mathrm{MeC}$ crystals, which moreover are too small in order to provide a sufficiently large volume for coherent scattering of X-rays that is necessary for the observation of diffraction interferences.

In summary, only for high metal content films nanocrystalline carbides are observed. No crystalline phases can be identified for low metal content films. It seems that the excess of carbon limits the growth of crystalline carbide grains during deposition [52]. Neither 
crystalline metal nor graphitic phases are observed. The latter finding is in agreement with the Raman analysis.

\subsection{Film morphology}

A multilayer structure is observed for a-C:VC $(21$ at.\% $\mathrm{V})$ in the cross-sectional TEM images of Figure 6. In total, five regions are found. Three of them exhibit an ordered multilayer pattern with a period of $(5 \pm 1) \mathrm{nm}$. In the other two this periodicity is apparently lost (Figure 6a). The observed layer sequence is correlated with the different compositional ranges observed in RBS (see Figure 2). High-resolution imaging was applied for detailed analysis of the ordered and non-ordered regions of a-C:CV (21 at.\% V) in Figure 6b. The two different regions are labeled as 1 and 2. The FFT analysis of the non-ordered region 1 reveals only one lattice plane distance of $2.4 \AA$ (Figure 6c). This distance is attributed to the (111) planes of $f c c$-VC phase, revealing VC nanocrystals embedded in the a-C matrix.

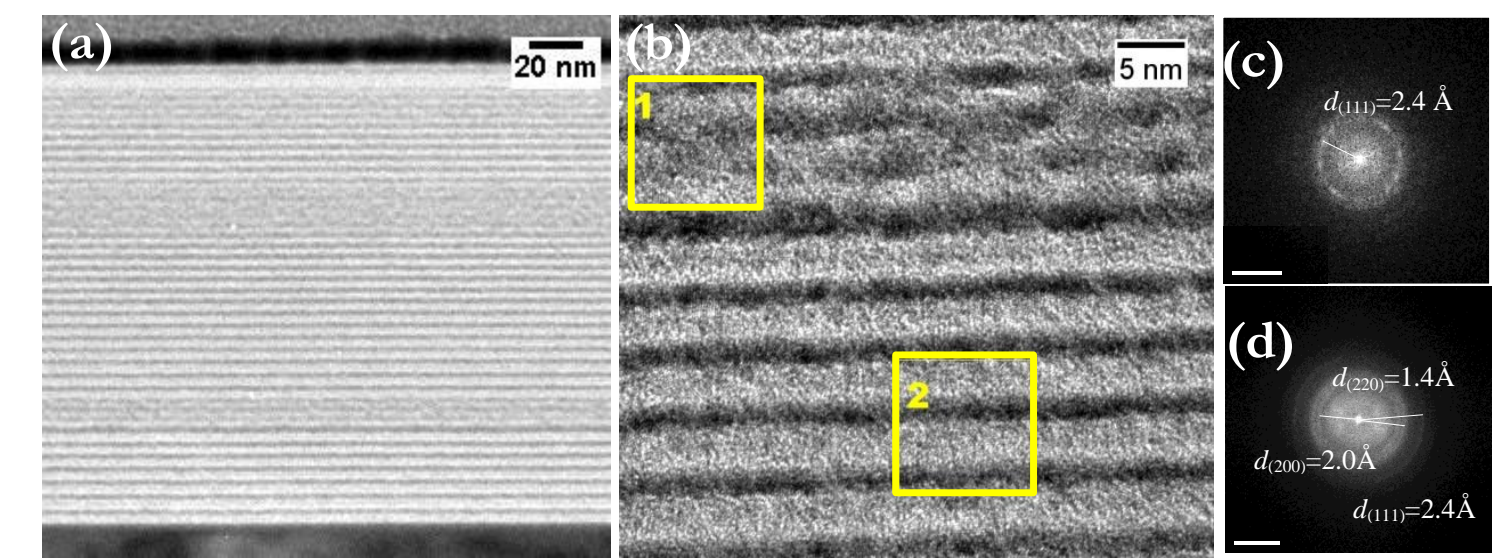

Figure 6: HRTEM analysis of a-C:VC (21 at.\%V) deposited on Inconel. (a) Overview image, (b) high-resolution image of a representative sample section, (c) and (d) FFTs analysis of selected regions 1 and 2 of (b), respectively.

The self-organized multi-layers of region 2 are composed of alternating dark contrast carbiderich $(\sim 1-1.5 \mathrm{~nm}$ thick $)$ and bright contrast carbide-depleted $(\sim 3-4 \mathrm{~nm})$ nanolayers. The FFT of this region shows three circular intensity maxima (Figure 6d). The corresponding lattice plane distances of 1.4, 2.0 and $2.4 \AA$ are in very good agreement with those of $f c c$-VC [53].

The multilayer structure observed for a-C:VC (21 at.\% V) cannot be associated with the sample rotation during the deposition process as the periodicity of the layers does not match the rotation speed of the substrate holder and the sample holder orientation has no effect on microstructure evolution. Moreover, it is not correlated with the pulse sequence during the deposition. Therefore, the formation of the layered structure is attributed to a self-organization process already reported in the literature [54-57]. 


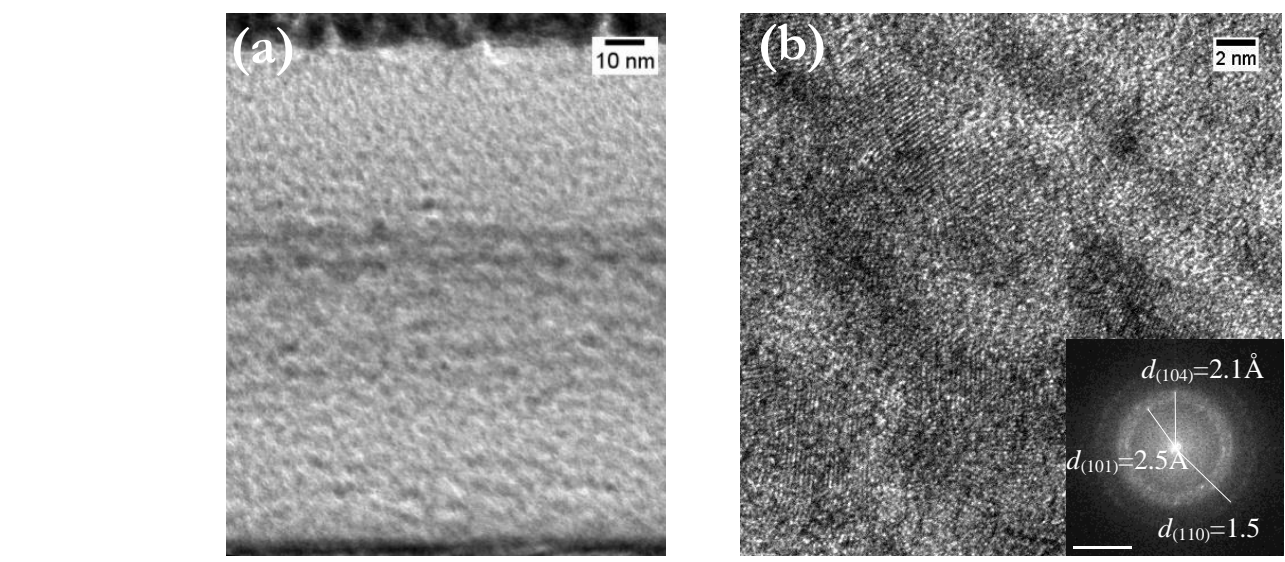

Figure 7: HRTEM analysis of a-C:MoC (19 at.\%Mo) deposited on Inconel. (a) cross-section image, (b) high-resolution image of an illustrative sample area, with FFTs analysis of (b).

HRTEM images of the a-C:MoC (19 at.\% Mo) sample (Figure 7a) reveal three different regions, two metal carbide depleted (brighter areas) and one metal carbide-rich (darker area). This is consistent with the three regions identified by RBS analysis (Figure 2).

High-resolution TEM images reveal molybdenum carbide nanoparticles embedded in a carbon matrix (Figure 7b). The FFT analysis gives three circular intensity maxima associated with lattice parameters of 1.5, 2.1 and $2.5 \AA$ (inset Figure 7b). These interplanar distances are in good agreement with those of the (110), (103) and (101) crystal planes of $\gamma$-MoC. Based on the slightly better agreement of the lattice plane distances and the thermodynamics the carbide phase of this nanocomposite is attributed to $\gamma-\mathrm{MoC}$ and not to $\beta-\mathrm{Mo}_{2} \mathrm{C}$.

In summary, the comprehensive characterization gives a consistent scenario of the microstructure evolution of the nanocomposites under study. The formation of nanocomposites structures of dispersed metal carbide crystals embedded in amorphous carbon was confirmed by Raman, XRD and TEM for the samples with high metal content. The phase structure is thermodynamically driven by the immiscibility of the phases which lead to a phase separation. In addition to the generally observed phase separation, a spontaneous formation of ordered multilayers for the case of the a-C:VC $(21 \mathrm{at} . \% \mathrm{~V})$ thin film is found.

This complete microstructural characterization of the thin films will be applied for additional information required in the simulations of the optical properties in the following section. The variety of obtained microstructures and the comprehensive characterization provides the basis for a complete simulation of the optical properties of the a-C:MeC nanocomposite solar absorber layers.

\subsection{Optical properties of a-C:VC and a-C:MoC films}

In order to simulate the optical properties of heterogeneous materials, it is crucial to find a proper EMA which accurately describes the combined effect of the nanocomposite 
components. Therefore, the measured reflectance spectrum of a selected nanocomposite film, namely a-C:VC (21 at.\% V ), was compared to simulated spectra obtained by using MaxwellGarnett (MG) [47], Bruggeman (BRU) [48], and Bergman (BER) [49] models (Figure 8).

In the simulations, the amorphous carbon was considered to form the matrix material. The strong dependence of the a-C optical constants on the ion energy, the substrate temperature, the plasma density and the $s p^{3}$ fraction, make their correct estimation a complex issue. Hence, the optical constants of the a-C matrix were estimated from spectroscopic ellipsometry (SE) measurements performed on the a-C reference sample. For an a-C deposited with FCVA with $s p^{3}$ content $\sim 20 \%$, Ferrari et al [46] estimated a density of $2.1 \mathrm{~g} \cdot \mathrm{cm}^{-3}$.

No metallic phase was experimentally found in any of the single layers (see section 3.3 and 3.4). Therefore, the $\mathrm{MeC}$ was considered to be the particle component of the nanocomposite. The metal atomic percentage obtained experimentally by RBS-NRA was converted into volume fraction (VF) of metal carbide using the densities of the individual components. $\mathrm{MeC}$ optical constants and density values of 5.6 and $9.5 \mathrm{~g} \cdot \mathrm{cm}^{-3}$ for $\mathrm{VC}$ and $\gamma$-MoC were obtained from literature data $[11,58,59]$.

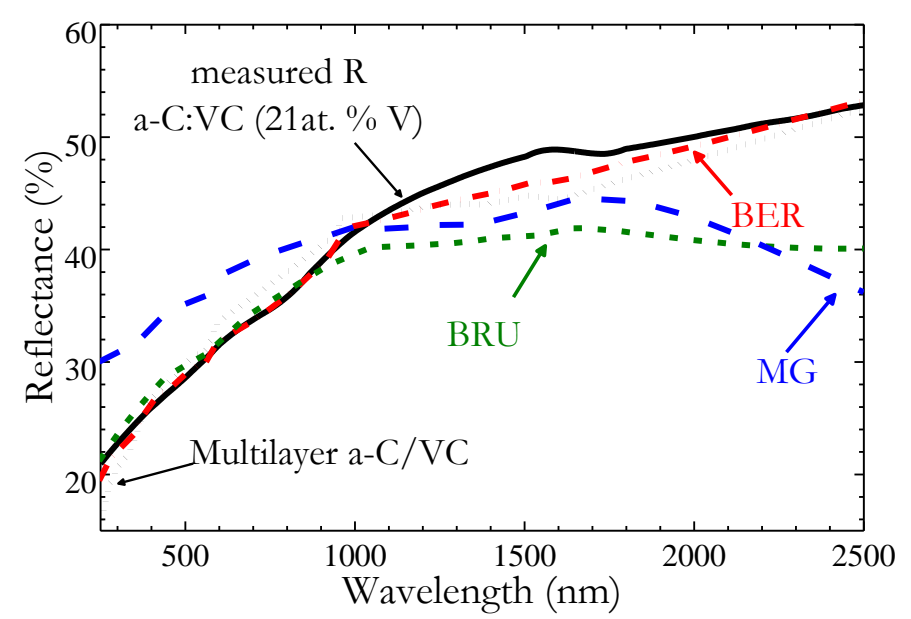

Figure 8: Comparison of measured reflectance spectrum of a-C:VC (21 at.\%), (solid black line) with simulation data obtained for the same composition employing Maxwell-Garnett (MG), Bruggeman (BRU) and Bergman (BER) effective medium approximations, with the equivalent $41.3 \%$ volume fraction of $\mathrm{VC}$, and 42 multilayer structure alternating between a-C (5nm) and VC (4nm) nanolayers.

The comparison of the simulated and measured spectra reveals that Bergman model properly describes the experimental reflectance of a-C:VC $(21$ at.\% $\mathrm{V})$ in the whole wavelength range (Figure 8). These significant differences of the three EMAs are also reflected in the solar absorptance (Table 3).

Table 3: Solar absorptance of deposited a-C:VC (21at.\% V) sample compared with the calculated solar absorptance for the equivalent simulated sample by Maxwell-Garnett, Bruggeman and Bergman EMA theories and with the multilayer a-C/VC.

\begin{tabular}{lc}
\hline EMA model & Solar absorptance (\%) \\
\hline Maxwell- Garnett (MG) & $61.3 \pm 0.1$ \\
Bruggeman (BRU) & $65.3 \pm 0.1$ \\
Bergman (BER) & $63.8 \pm 0.1$ \\
Multilayer a-C / VC & $63.7 \pm 0.1$ \\
\hline
\end{tabular}


Maxwell-Garnett overestimates the experimental reflectance by up to $10 \%$ in the visible and underestimates it by up $15 \%$ in the near infrared spectral range, giving the largest deviation of the simulated $\alpha(\Delta \alpha=-3.0 \%)$ from all the EMAs employed. MG approximation is only applicable to systems with volume fraction below $30 \%$ without percolation. As the volume fraction of the carbide particles in the a-C:VC (21 at.\% V) is $41 \%$, a high degree of percolation is expected, accounting for the strong deviation of MG simulation shown in Figure 8 .

Bruggeman theory is adequate for large particle volume fraction and percolated systems. It is the most commonly used approximation as it only requires the particle volume fraction as simulation parameter. However, this approach does not properly simulates the experimental reflectance spectra for a-C:VC films. This model provided an excellent agreement for the visible range but underestimated the reflectance in the near-infrared range. This results in small deviation of solar absorptance $(\Delta \alpha=+1.0 \%)$, but from deviations in the IR range one could expect misleading thermal emittance values.

As mentioned above, Bergman gives the best agreement between the simulated and experimental spectra in the whole wavelength range, with the most accurate value of solar absorptance $(\Delta \alpha=-0.5 \%)$. A tentative explanation for this finding is that this EMA incorporates the shape and degree of percolation of the embedded nanoparticles as additional parameters to the simulation. BER formula is described in equation (7).

$$
\varepsilon_{e f f}=\varepsilon_{M}\left(1-f \int_{0}^{1} \frac{g(n, f)}{t-n} d n\right) \text { with } \quad t=\frac{\varepsilon_{M}}{\varepsilon_{M}-\varepsilon_{P}}
$$

Where $\varepsilon_{p}$ and $\varepsilon_{M}$ are the particles and matrix material dielectric functions respectively, and $\varepsilon_{e f f}$ the effective dielectric function of the nanocomposite, $t$ is a combined dielectric property (takes real values between 0 and 1$), f$ is the particle volume fraction. $g(n, f)$ is a function called spectral density as expressed by equation (8):

$$
g(n, f)=g_{0}(f) \delta(n)+g_{\text {cont }}(n, f)
$$

Consequently, the fitting procedure involves three parameters: volume fraction, percolation strength, and $n$, an arbitrary number of points that are used to define the shape of $g(n, f)$ by a cubic spline interpolation. With the BER representation, the average dielectric functions $\varepsilon_{\text {eff }}$ of the composite material were calculated from the individual components ( $\varepsilon_{M}$ and $\left.\varepsilon_{P}\right)$.

In order to clarify whether the multilayer structure of a-C:VC (21 at.\% V) observed in the HRTEM images affects the optical film properties, a multilayered structure was also simulated. It is formed of 21 bilayers of pure metal carbide and pure amorphous carbon of 4 
and $5 \mathrm{~nm}$ thickness, respectively, corresponding to the measured carbide volume fraction $(41 \%$ VF). No significant differences were observed between the multilayer reflectance simulation $(\alpha=63.7 \%)$ and BER simulation as shown in Table 3. This result implies that the film growth mode (self-organized layered structure or dispersed carbide nanoparticles embedded in a-C matrix) does not affect the optical properties. A similar result has been observed in solar selective coatings based on silicon nitride cermets [60].

Once the appropriateness of the Bergman model to simulate a-C:MeC composites is demonstrated, this EMA was used to simulate reflectance spectra and solar absorptance for the all studied nanocomposite samples (Figure 9).

The function $g(n, f)$ cannot be computed analytically for real systems since the topology is too complicated or not even known. BER representation parameters were calculated by adjusting $g(n, f)$ to experimental data, and the fitting parameters are further detailed in the supplementary data. The effective dielectric function obtained provides additional information related to the shape of the nanoparticles and their degree of percolation. BER theory is especially interesting when the microstructure plays a significant role in the optical properties of the heterogeneous material.[61]
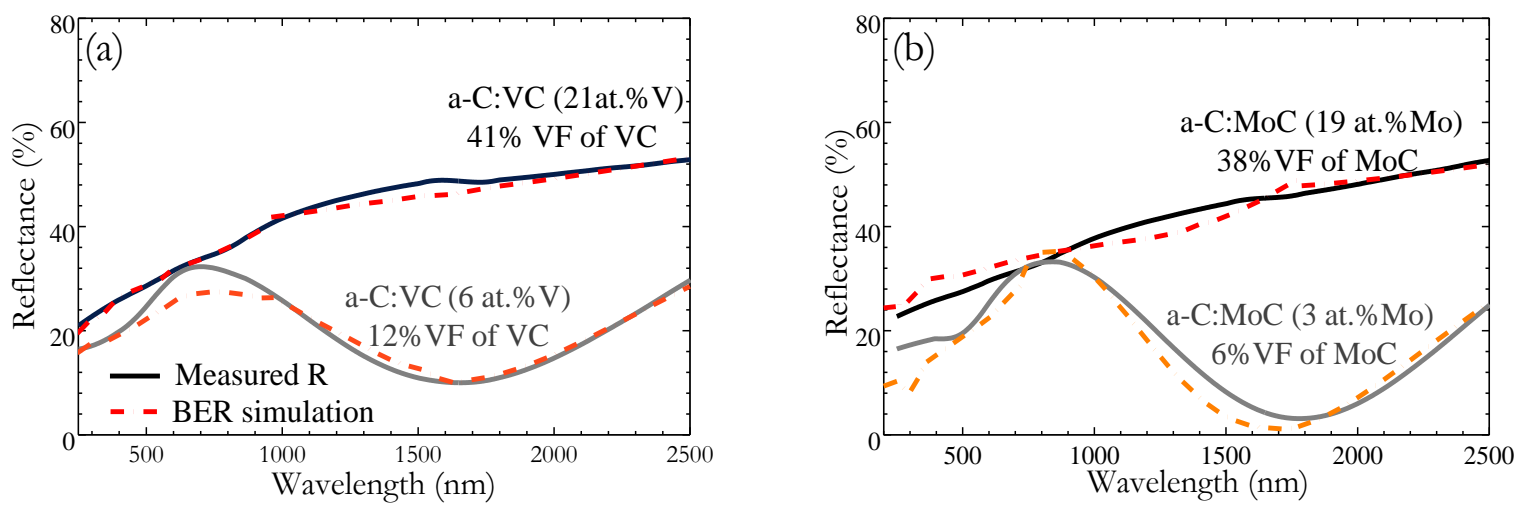

Figure 9: Comparison of measured and simulated reflectance spectra of (a) a-C:VC and (b) a-C:MoC for samples with higher and lower metal content. a-C:VC $(21 \mathrm{at} . \% \mathrm{~V})$ has been represented in Figure 8, but is plotted again here for comparison. Solid lines represent the measured reflectance spectra and the dash-dot is the simulated reflectance following Bergman EMA.

An excellent agreement was obtained for all the studied systems, independently of the metal carbide content. Bergman model allows extracting percolation behaviour according to the metal content in the samples. In the case of nanocomposites with higher metal content, the percolation strength $\left(g_{0}\right)$ necessary to ensure a correct fit between measured and simulated spectra was $\sim 1$. This is an indication of interconnection of the embedded carbide particles, pointing to a metallic character of the a-C:MeC(h) films. On the contrary, samples with low metal content, exhibit no percolation $\left(g_{0}(f)=0\right)$. This is characteristic for a dielectric or insulating behaviour, caused by the missing connection between the carbide particles. 
On the basis of the Bergman representation, the solar absorptance $(\alpha)$ was computed as a function of carbide volume fraction and thickness (Figure 10) for samples on Inconel substrate.
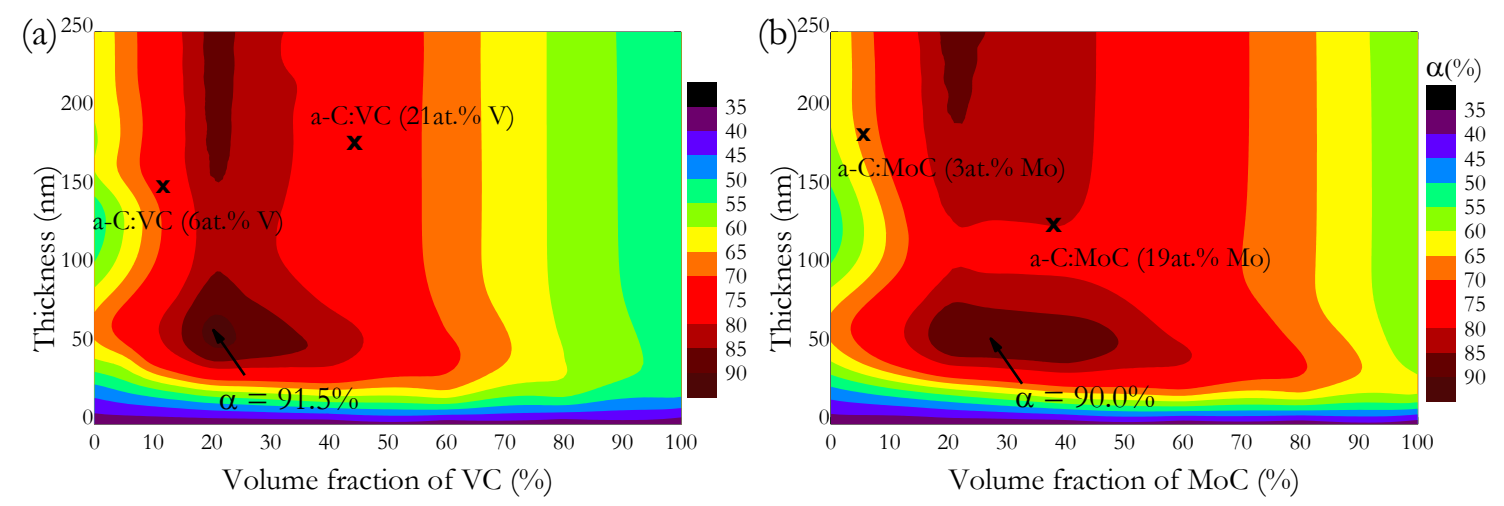

Figure 10: Solar absorptance for different thicknesses and volume fraction values of (a) a- C:VC and (b) a-C:MoC. The maximum absorptance for each a-C:MeC type obtained from simulation, as well as the experimentally deposited samples, are also included in the graphs.

In both systems, the absorptance maximum was found for a volume fraction in the range of 15 to $40 \% \mathrm{VF}$. By variation of the thickness, $\alpha$ increases from $36 \%$ (absorptance of the inconel substrate) until it reaches a maximum for a thickness of 50 to $70 \mathrm{~nm}$. The maximum $\alpha$ is 91.5 $\pm 0.5 \%$ for a $62 \mathrm{~nm}$ thick a-C:VC single layer with $19 \%$ volume fraction of VC (equivalent to 9.7 at.\% of $\mathrm{V}$ ) on Inconel substrate In the case of the a-C:MoC system, a maximum absorptance of $90 \pm 0.5 \%$ was found for a film with thickness of $55 \mathrm{~nm}$ and $28 \% \mathrm{VF}$ of MoC (14 at.\% Mo). For thicknesses higher than $150 \mathrm{~nm}$, the absorptance increases again, reaching a maximum constant value similar to the one in the thinner range. However, increasing the absorber layer thickness can negatively affect to interlayer adherence and/or increase of thermal emittance in a complete solar selective coating. Hence, the thickness considered for further simulations was in the 50-70 $\mathrm{nm}$ range.

Using the knowledge gained from the individual absorber layer study, a complete solar selective stack structure (AR/a-C:MeC/IR) was simulated (Figure 11). $\mathrm{Al}_{2} \mathrm{O}_{3}$ with $\sim 45 \mathrm{~nm}$ thickness was selected as AR layer due its high-temperature resistance and inertness in relation to the environment. For the absorber layer, the optimized thickness and volume fraction values for a-C:VC and a-C:MoC were adopted. A $400 \mathrm{~nm}$ TiN infrared reflectance layer was also added between the substrate and the $\mathrm{a}-\mathrm{C}: \mathrm{MeC}$ in order to reduce the emittance in the infrared part of the spectrum. 


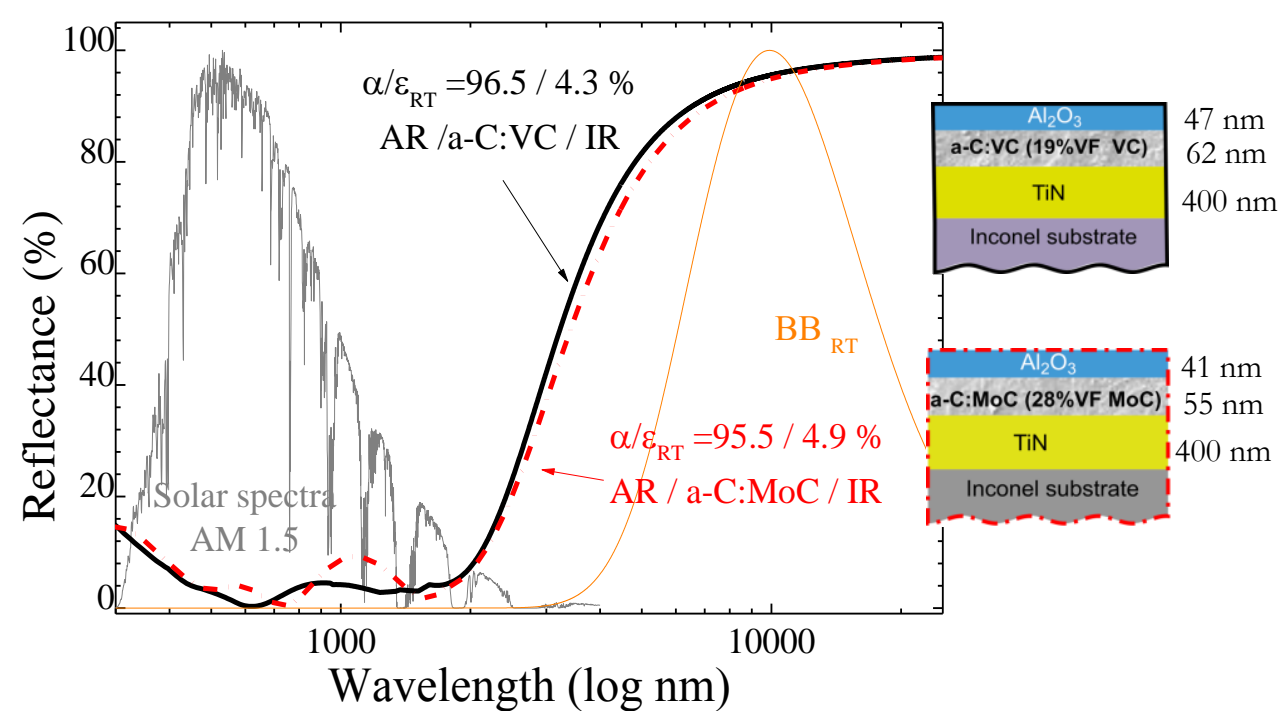

Figure 11: Simulated reflectance spectra of $\mathrm{Al}_{2} \mathrm{O}_{3} / \mathrm{a}-\mathrm{C}: \mathrm{VC}(19 \% \mathrm{VF} \mathrm{VC}) / \mathrm{TiN}$ and $\mathrm{Al}_{2} \mathrm{O}_{3} / \mathrm{a}-\mathrm{C}: \mathrm{MoC}(28 \% \mathrm{VF} \mathrm{MoC}) / \mathrm{TiN}$ of the optimized solar selective coatings. The solar spectrum (AM1.5) and the black body radiation at $25^{\circ} \mathrm{C}$ (Planck's law) are included.

The solar selective coatings for both the AR / a-C:VC / IR and AR / a-C:MoC / IR coatings, provide a selectivity ratio $\alpha / \mathcal{E}_{R T}=96.5 / 4.3$ at RT. At $600^{\circ} \mathrm{C}$, the emittance increases up to $14 \%$ and $15 \%$ for the simulated a-C:VC and a-C:MoC based multilayer stack, respectively. Those values are among the best performing reported for simulated layer stacks using a-C:MeC $(\alpha=$ $91.0 \%$ and $\varepsilon_{100^{\circ} \mathrm{C}}=11 \%$ for a-C: $\mathrm{TiC} / \mathrm{SiO}_{2}$ on $\mathrm{Cu}$ substrate) $[27,34]$ as absorber layer. The optimized values for cermets as absorber were $\alpha=95.0 \%$ with $\varepsilon_{300^{\circ} \mathrm{C}}$ of $9 \%$ for a graded WAlN $[62,63]$.

\section{Conclusions}

Solar selective surfaces have been designed based on carbon transition metal carbide absorber layers. Filtered cathodic arc was used for depositing a-C:VC and a-C:MoC films. For higher metal content ( $\sim 21$ at.\% V and $\sim 19$ at.\% Mo) film microstructure consists of metal carbides particles embedded in an amorphous carbon matrix for samples deposited with higher metal content. In the case of a-C:VC films, a self-organized arrangement of multilayers was observed.

Reflectance spectra were simulated employing different EMA models using experimentally measured optical constants for a-C. An excellent agreement between simulated and measured reflectance for single a-C:MeC layers was only achieved in the whole wavelength range using a Bergman approach. This approach requires a detailed microstructural analysis for an accurate optical simulation in inhomogeneous materials.

Using Bergman EMA model, the maximum solar absorptance of $91.5 \pm 0.5 \%$ is obtained for the a-C:VC single layer on Inconel substrate with a film $62 \mathrm{~nm}$ thickness and $19 \%$ volume 
fraction of VC (equivalent to 9.7 at.\% of V). In the case of the a-C:MoC system, it is possible to reach an absorptance of $90 \pm 0.5 \%$ for a film with thickness of $55 \mathrm{~nm}$ and $28 \%$ volume fraction of MoC (14 at.\% of metal Mo).

The simulation of a complete solar selective surface and antireflective and IR mirror layers leads to a solar absorptance higher than $96 \%$ with a thermal emittance at room temperature below $5 \%$ and $15 \%$ at $600^{\circ} \mathrm{C}$. This demonstrates the potential of a-C:MeC as an absorber layer material for high-temperature applications. Further experimental studies should be carried out on such solar selective surfaces in order to test the validity of the predictions of this study on optical properties at RT and high temperature as well as to test thermal and environmental stability.

\section{Acknowledgments}

This project was partially supported by H2020 RISE project "Framework of Innovation for Engineering of New Durable Solar Surfaces (FRIENDS ${ }^{2}$, GA-645725)" and Centro para el Desarrollo Tecnológico Industrial (CDTI) project "INvestigación y DEsarrollo de recubrimientos SOLares selectivos de alta temperatura obtenidos mediante técnicas PVD (INDESOL)" IDI-20130896.

Support by the Ion Beam Center (IBC, Helmholtz-Zentrum Dresden-Rossendorf) is gratefully acknowledged. The authors would like to thank R. Bonet and J. Caro (Centre Tecnologic Manresa) for their help on the deposition of the samples; R. Wenisch, F. Lungwitz, E. Schumann and R. Heller (Helmholtz-Zentrum Dresden - Rossendorf) for assistance during the use of IBA, ellipsometry, spectrophotometry, and Raman techniques at the IBC; A. Mayoral (Laboratorio de Microscopies Avanzadas from Instituto de Nanociencia de Aragón) for offering access to their instruments and expertise with TEM measurement, and $\mathrm{M}^{\mathrm{a}}$. C. López Santos (Instituto Ciencia y Tecnología de Materiales de Sevilla) for the XRD service.

Any opinions, findings and conclusions or recommendations expressed in this material are those of the authors and do not necessarily reflect those of the host institutions or funders.

\section{$6 \quad$ References}

[1] L.A. Weinstein, J. Loomis, B.B. Bhatia, D.M. Bierman, E.N. Wang, G.G. Chen, Concentrating Solar Power, Chem. Rev. 115 (2015) 12797.

[2] Q. Zhang, Recent progress in high-temperature solar selective coatings, Sol. Energy Mater. Sol. Cells. 62 (2000) 63-74. 
[3] N. Selvakumar, H.C. Barshilia, Review of physical vapor deposited (PVD) spectrally selective coatings for mid- and high-temperature solar thermal applications, Sol. Energy Mater. Sol. Cells. 98 (2012) $1-23$

[4] S. Wijewardane, D.Y. Goswami, A review on surface control of thermal radiation by paints and coatings for new energy applications, Renew. Sustain. Energy Rev. 16 (2012) 1863-1873.

[5] C. Zou, L. Huang, J. Wang, S. Xue, Effects of antireflection layers on the optical and thermal stability properties of a spectrally selective CrAlN-CrAlON based tandem absorber, Sol. Energy Mater. Sol. Cells. 137 (2015) 243-252.

[6] M.G. Hutchins, Spectrally Selective Solar Absorber Coatings, Appl. Energy. 5 (1979) 251.

[7] P. Oelhafen, A. Schüler, Nanostructured materials for solar energy conversion, Sol. Energy. 79 (2005) 110-121.

[8] K.T. Roro, N. Tile, A. Forbes, Preparation and characterization of carbon/nickel oxide nanocomposite coatings for solar absorber applications, Appl. Surf. Sci. 258 (2012) 7174-7180.

[9] G.L. Harding, Absorptance and emittance of metal carbide selective surfaces sputter deposited onto glass tubes, Sol. Energy Mater. 2 (1980) 469-481.

[10] S. Logothetidis, Optical and electronic properties of amorphous carbon materials, Diam. Relat. Mater. 12 (2003) 141-150.

[11] H.O. Pierson, Handbook of refractory carbides and nitrides: properties, characteristics, processing, and applications, Noyes Publications, Westwood,New Yersey USA, 1996.

[12] U. Jansson, E. Lewin, Sputter deposition of transition-metal carbide films - A critical review from a chemical perspective, Thin Solid Films. 536 (2013) 1-24.

[13] M. Berndt, G. Abrasonis, G.J. Kovács, M. Krause, F. Munnik, R. Heller, et al., Bulk diffusion induced structural modifications of carbon-transition metal nanocomposite films, J. Appl. Phys. 109 (2011) 063503-11.

[14] S. Zhang, X.L. Bui, X. Li, Thermal stability and oxidation properties of magnetron sputtered diamond-like carbon and its nanocomposite coatings, Diam. Relat. Mater. 15 (2006) 972-976.

[15] K. Sedláčková, P. Lobotka, I. Vávra, G. Radnóczi, Structural, electrical and magnetic properties of carbon-nickel composite thin films, Carbon N. Y. 43 (2005) 2192-2198.

[16] G.J. Kovács, G. Sáfrán, O. Geszti, T. Ujvári, I. Bertóti, G. Radnóczi, Structure and mechanical properties of carbon-nickel and $\mathrm{CN}_{\mathrm{x}}$-nickel nanocomposite films, Surf. Coatings Technol. 180 (2004) $331-334$.

[17] G. Abrasonis, A.C. Scheinost, S. Zhou, R. Torres, R. Gago, I. Jiménez, et al., X-ray Spectroscopic and Magnetic Investigation of C:Ni Nanocomposite Films Grown by Ion Beam Cosputtering, J. Phys. Chem. C. 112 (2008) 12628-12637.

[18] K. Sedláčková, T. Ujvári, R. Grasin, P. Lobotka, I. Bertóti, G. Radnóczi, C-Ti nanocomposite thin films: Structure, mechanical and electrical properties, Vacuum. 82 (2007) 214-216.

[19] I. Gerhards, H. Stillrich, C. Ronning, H. Hofsäss, M. Seibt, Self-organized nanoscale multilayer growth in hyperthermal ion deposition, Phys. Rev. B. 70 (2004) 245418.

[20] R.J. Narayan, Pulsed laser deposition of functionally gradient diamondlike carbon-metal nanocomposites, Diam. Relat. Mater. 14 (2005) 1319-1330.

[21] G.L. Harding, Sputtered metal carbide solar-selective absorbing surfaces, J. Vac. Sci. Technol. 13 (1976) 1070.

[22] G.L. Harding, Magnetron-sputtered metal carbide solar selective absorbing surfaces, J. Vac. Sci. Technol. 16 (1979) 857.

[23] R. Gampp, P. Gantenbein, Y. Kuster, P. Reimann, R. Steiner, P. Oelhafen, et al., Characterization of a-C:H/W and a-C:H/Cr solar selective absorber coatings, Proc. SPIE. 2255 (1994) 92-106. 
[24] R. Gampp, P. Gantenbein, Preparation and characterization of chromium containing amorphous hydrogenated carbon films (a-C:H/Cr), Proc. MRS. 388 (1995) 305-310.

[25] R. Gampp, P. Oelhafen, P. Gantenbein, S. Brunold, U. Frei, Accelerated aging tests of chromium containing amorphous hydrogenated carbon coatings for solar collectors, Sol. Energy Mater. Sol. Cells. 54 (1998) 369-377.

[26] Y. Yin, D.R. Mckenzie, W.D. Mcfall, Cathodic arc deposition of solar thermal selective surfaces, Sol. Energy Mater. Sol. Cells. 44 (1996) 69-78.

[27] A. Schüler, I.R. Videnovic, P. Oelhafen, S. Brunold, Titanium-containing amorphous hydrogenated silicon carbon films (a-Si:C:H/Ti) for durable solar absorber coatings, Sol. Energy Mater. Sol. Cells. 69 (2001) 271-284.

[28] W. Theiss, The use of effective medium theories in optical spectroscopy, Adv. Solid State Phys. 33 (1994) 149-176.

[29] J.C. Maxwell-Garnett, Colours in Metal Glasses, in Metallic Films, and in Metallic Solutions. II, Philos. Trans. R. Soc. A Math. Phys. Eng. Sci. 205 (1906) 237-288.

[30] D. Bruggeman, Calculation of various physics constants in heterogenous substances I Dielectricity constants and conductivity of mixed bodies from isotropic substances, Ann. Phys. 24 (1935) 636-664.

[31] D. Bergman, Dielectric - Constant if a Composite material - Problem in classical Physicis, Phys. Reports Rev. Sect. Phys. Lett. 43 (1978) 378-407.

[32] A. Anders, Energetic deposition using filtered cathodic arc plasmas, Vacuum. 67 (2002) $673-$ 686.

[33] W. Theiss, CODE Manual. Optical Spectrum Simulation, (2007). http://www.wtheiss.com/ (accessed November 4, 2015).

[34] D.P. Gruber, G. Engel, H. Sormann, A. Schüler, W. Papousek, Modeling the absorption behavior of solar thermal collector coatings utilizing graded alpha-C:H/TiC layers., Appl. Opt. 48 (2009) 1514-9.

[35] A. Pardo, J.G. Buijnsters, J.L. Endrino, C. Gómez-Aleixandre, G. Abrasonis, R. Bonet, et al., Effect of the metal concentration on the structural, mechanical and tribological properties of selforganized a-C:Cu hard nanocomposite coatings, Appl. Surf. Sci. 280 (2013) 791-798.

[36] M.M.M. Bilek, R.N. Tarrant, D.R. McKenzie, S.H.N. Lim, D.G. McCulloch, Control of stress and microstructure in cathodic arc deposited films, IEEE Trans. Plasma Sci. 31 (2003) 939-944.

[37] J.R. Tesmer, M. Nastasi, Handbook of modern ion beam materials analysis, Materials Research Society, Pittsburg, Pennsylvania, 1995.

[38] G. Abrasonis, M. Krause, A. Mücklich, K. Sedlac ková, G. Radnóczi, U. Kreissig, et al., Growth regimes and metal enhanced 6-fold ring clustering of carbon in carbon-nickel composite thin films, Carbon N. Y. 45 (2007) 2995-3006.

[39] M. Krause, A. Mücklich, C. Wilde, M. Vinnichenko, S. Gemming, G. Abrasonis, Structure , Optical and Mechanical Properties of Direct Current Magnetron Sputtered Carbon: Vanadium Nanocomposite Thin Films, Nanosci. Nanotechnol. Lett. 5 (2013) 94-100.

[40] B.D. Cullity, S.R. Stock, Elements of X-ray diffraction, Prentice-Hall, Massachussets, 2001.

[41] ASTM, ASTM G173: Standard Tables for Reference Solar Spectral Irradiance at Air Mass 1.5: Direct Normal and Hemispherical for a 37 Degree Tilted Surface, US, 2008.

[42] J.A. Duffie, W.A. Beckman, Solar engineering of thermal processes., 3rd ed., John Wiley \& Sons, New York, 2005.

[43] EU Standard, N. Europeenne, UNE-EN 673 Determinación del coeficiente de transmisión térmica, Europe, 2011. 
[44] Y. Wang, M. Nastasi, Handbook Modern Ion Beam Materials Analysis, 2nd edition, Cambridge University Press, 2010.

[45] T. Koide, T. Shidara, H. Fukutani, A. Fujimori, S. Otani, Y. Ishizawa, Optical Constants of TiC 0.95 , VC 0.86 and NbC 0.93 from 0.8 to 80 eV, Jpn. J. Appl. Phys. 32 (1993) 1130-1134.

[46] A.C. Ferrari, B. Kleinsorge, G. Adamopoulos, J. Robertson, W.I. Milne, V. Stoloja, et al., Determination of bonding in amorphous carbons by electron energy loss spectroscopy , Raman scattering and X-ray reflecectivity, J. Non. Cryst. Solids. 266-269 (2000) 765-768.

[47] A. Ferrari, J. Robertson, Interpretation of Raman spectra of disordered and amorphous carbon, Phys. Rev. B. 61 (2000) 14095-14107.

[48] M. Berndt, M. Krause, G. Abrasonis, A. Mücklich, F. Munnik, A. Kolitsch, et al., Morphology and Structure of C:Co, C:V, and C:Cu Nanocomposite Films, Plasma Process. Polym. 6 (2009) S902S906

[49] ICDD Powder Diffraction Data files: 03-065-7885 for fcc VC, 00-008-0384 for hex-MoC, 00015-0457 for hep $\mathrm{Mo}_{2} \mathrm{C}$.

[50] E. Portolan, C.L.G. Amorim, G.V. Soares, C. Aguzzoli, C.A. Perottoni, I.J.R. Baumvol, et al., Carbon occupancy of interstitial sites in vanadium carbide films deposited by direct current reactive magnetron sputtering, Thin Solid Films. 517 (2009) 6493-6496.

[51] T. Massalski, Binary alloy phase diagrams, ASM International, Materials Park, Ohio, 1990. http://www.worldcat.org/title/binary-alloy-phase-diagrams/oclc/22623894 (accessed November 4, 2013).

[52] G. Abrasonis, M. Berndt, M. Krause, K. Kuepper, F. Munnik, A. Kolitsch, et al., Soft X-ray Absorption and Emission Spectroscopic Investigation of Carbon and Carbon: Transition Metal Composite Films, J. Phys. Chem. C. 112 (2008) 17161-17170.

[53] M. Berndt, M. Krause, G. Abrasonis, A. Mücklich, F. Munnik, A. Kolitsch, et al., Morphology and Structure of C:Co, C:V, and C:Cu Nanocomposite Films, Plasma Process. Polym. 6 (2009) S902S906.

[54] G. Abrasonis, G.J. Kovács, M.D. Tucker, R. Heller, M. Krause, M.C. Guenette, et al., Sculpting nanoscale precipitation patterns in nanocomposite thin films via hyperthermal ion deposition, Appl. Phys. Lett. 97 (2010) 163108.

[55] W.-Y. Wu, J.-M. Ting, Self-assembled alternating nano-scaled layers of carbon and metal, Chem. Phys. Lett. 388 (2004) 312-315.

[56] M. Krause, M. Buljan, M. Arndt, M. Wolfhard, M. Fritzsche, S. Facsko, et al., Compositionally modulated ripples during composite film growth: Three-dimensional pattern formation at the nanoscale, Phys. Rev. B. 89 (2014) 085418.

[57] S. Anders, D.L. Callahan, G.M. Pharr, T.Y. Tsui, C. Singh Bhatia, Multilayers of amorphous carbon prepared by cathodic arc deposition, Surf. Coatings Technol. 94 (1997) 189-194.

[58] H. Nowotny, E. Parthé, R. Kieffer, F. Benesovsky, Das Dreistoffsystem: MolybdänSilizium-Kohlenstoff, Phys. Anorg. Und Anal. Chemie. 65 (1954) 255.

[59] F. Modine, T. Haywood, C. Allison, Optical and electrical properties of single-crystalline zirconium carbide, Phys. Rev. B. 32 (1985) 7743-7747.

[60] E. Céspedes, M. Wirz, J.A. Sánchez-García, L. Alvarez-Fraga, R. Escobar-Galindo, C. Prieto, Novel $\mathrm{Mo}-\mathrm{Si}_{3} \mathrm{~N}_{4}$ based selective coating for high temperature concentrating solar power applications, Sol. Energy Mater. Sol. Cells. 122 (2014) 217-225.

[61] G. a Niklasson, C.G. Granqvist, Optical properties and solar selectivity of coevaporated CoAl2O3 composite films, J. Appl. Phys. 55 (1984) 3382-3410.

[62] Q. Zhang, Optimizing analysis of W-AlN cermet solar absorbing coatings, J. Phys. D. Appl. Phys. 34 (2001) 3113-3120. 
[63] J.F. Li, L.Z. Guo, Computer Simulation on $\mathrm{Mo} / \mathrm{Al}_{2} \mathrm{O}_{3}$ Solar Energy Selective Absorbing Coating, Adv. Mater. Res. 189-193 (2011) 66-69. 


\title{
Advanced characterisation and optical simulation for the design of solar selective coatings based on carbon: transition metal carbide nanocomposites
}

\author{
I. Heras ${ }^{1}$, M. Krause ${ }^{2}$, G. Abrasonis ${ }^{2}$, A. Pardo ${ }^{3}$, J. L. Endrino ${ }^{4}$, E. Guillén ${ }^{1}$, R. Escobar Galindo ${ }^{1^{*}}$ \\ ${ }^{1}$ Abengoa Research S. L., Abengoa, Seville, Campus Palmas Altas 41014, Spain. \\ ${ }^{2}$ Helmholtz-Zentrum Dresden-Rossendorf, Dresden, Germany. \\ ${ }^{3}$ Centre Tecnologic de Manresa, Manresa, Spain \\ ${ }^{4}$ School of Aerospace, Transport and Manufacturing, Cranfield University, MK43z0AL Bedfordshire, UK.
}

\section{Supplementary data}

\section{Raman fitting}

The parameters extracted from Raman fitting are presented in Table S1.

Table S1: Experimental results from visible Raman spectroscopy of the deposited a-C:MeC thin films.

\begin{tabular}{cccccc}
\hline \multirow{2}{*}{ Sample } & \multicolumn{2}{c}{$\boldsymbol{D}$ band } & \multicolumn{2}{c}{$\boldsymbol{G}$ band } & \multirow{2}{*}{$\boldsymbol{I}_{\boldsymbol{D}}$} \\
\hline a-C & $1375 \pm 1$ & $236.5 \pm 0.5$ & $1544 \pm 1$ & $190.0 \pm 0.5$ & 0.40 \\
a-C:VC (21 at.\%V) & $1364 \pm 1$ & $238.0 \pm 0.5$ & $1545 \pm 1$ & $166.5 \pm 0.5$ & 0.81 \\
a-C:VC (6 at.\%V) & $1364 \pm 1$ & $227.0 \pm 0.5$ & $1539 \pm 1$ & $185.0 \pm 0.5$ & 0.56 \\
a-C:MoC(19 at.\%Mo) & $1363 \pm 1$ & $231.5 \pm 0.5$ & $1539 \pm 1$ & $173.0 \pm 0.5$ & 0.64 \\
a-C:MoC (3 at.\%Mo) & $1373 \pm 1$ & $223.5 \pm 0.5$ & $1543 \pm 1$ & $183.0 \pm 0.5$ & 0.50 \\
\hline
\end{tabular}

\section{Optical simulations. Effective medium approximations (EMA) theories.}

The complex refractive index of two-phase nanocomposites can be determined by a nontrivial mixing of the optical constants of the individual components by the so-called EMA theories. If the average size of the inhomogeneities or particles is much smaller than the wavelength of the incident radiation, the electric and magnetic fields are almost constant over this length [1], and hence, the composite behaves like quasi-homogeneous material.

These theories require knowing the optical constants of the embedded particles $\left(\varepsilon_{P}\right)$ and the host (matrix) material $\left(\varepsilon_{M}\right)$ to determine the effective dielectric function $\left(\varepsilon_{\text {eff }}\right)$ of the composite material. The particle size and distribution in the host matrix are assumed by the volume fraction $f$ as defined in equation (S.1).

$$
f=\frac{V_{\text {particles }}}{V_{\text {total }}}
$$

The simplest EMA theory is the Maxwell-Garnett (MG) theory. This theory considers that the inhomogeneous material has a separated grain structure [2] without particle interactions (no percolation). MG is only applicable to systems of low volume fraction of embedded particles $(f<0.3)$. The MG theory for identical spherical particles is defined in equation (S.2):

\footnotetext{
Corresponding author. Tel +34 649833 073; e-mail address: ramon.escobar@abengoa.com (R. EscobarGalindo)
} 


$$
\frac{\varepsilon_{e f f M G}-\varepsilon_{M}}{\varepsilon_{e f f M G}+2 \varepsilon_{M}}=f \frac{\varepsilon-\varepsilon_{M}}{\varepsilon+2 \varepsilon_{M}}
$$

The most commonly used EMA theory was proposed by Bruggeman (BRU) in 1935 [3]. It is applicable to aggregate microstructures where the host matrix and the distributed particles are hardly distinguishable. BRU theory has proven to be adequate for large particle volume fraction $(f \geq 0.3)$ and percolated systems. The BRU formula for spherical shaped particles is expressed in equation (S.3)

$$
(1-f) \frac{\varepsilon_{M}-\varepsilon_{e f f B R U}}{\varepsilon_{M}+2 \varepsilon_{e f f B R U}}+f \frac{\varepsilon-\varepsilon_{e f f B R U}}{\varepsilon+2 \varepsilon_{e f f B R U}}=0
$$

For very low volume fraction $(f<0.3)$ BRU is equivalent to MG theory, and no percolation is considered. Above his threshold, the embedded particles are assumed to be partially connected. Both MG and BRU theories have in common that the effective dielectric function does not depend explicitly on the size of the inhomogeneities. This is because the electric dipole term, which is proportional to particle volume, is retained in the series expansion of the amplitude of the electric field scattered by a single particle. However, important discrepancies between experimental and simulation results are found when only volume fraction is taken into account to describe the micro-topology of a nanocomposite material [4].

In 1978, Bergman [5] developed a theory where the microgeometry of the nanocomposite is taken into account by a spectral density function $g(n, f)$. Bergman representation (BER) is described by equation (S.4):

$$
\varepsilon_{e f f B E R}=\varepsilon_{M}\left(1-f(G(t)) \quad \text { with } \quad G(t)=\int_{0}^{1} \frac{g(n, f)}{t-n} d n\right.
$$

where $t$ is the reduced dielectric function, that takes real values between 0 and 1 , as equation (S.5):

$$
t=\frac{\varepsilon_{M}}{\varepsilon_{M}-\varepsilon_{P}}
$$

The function $g(n, f)$ holds details of the microgeometry. It is a real, non-negative function, normalized in the interval $[0,1]$ with an arbitrary number of points $(n)$ that are used to define the shape of the function by a cubic spline interpolation. Any microgeometry can be represented with a specific $g(n, f)$. However, it cannot be computed analytically for real systems since the topology is too complicated or not even known. If the system has some degree of connectivity between the embedded particles, it is useful to split the spectral density function in a diverging $\delta$ function and a continuous part [6] as defined in (S.6):

$$
g(n, f)=g_{0} \delta(n)+g_{\text {cont }}(n, f)
$$

where $g_{0}$ is the percolation strength that describes the metallic or dielectric behaviour of the nanocomposite. The parameter $g_{0}$ varies between 0 (no percolation) and 1 (total interconnection between the nanoparticles). BER representation parameters are difficult to determine and they are usually calculated by adjusting $g(n, f)$ to experimental data [7]. The effective dielectric function obtained provides additional information related to the shape of the nanoparticles and their degree of percolation. BER theory is especially interesting when 
the microstructure plays a significant role in the optical properties of the heterogeneous material [8].

The parameters extracted from the curve-fitting with Bergman EMA are presented in Table S2. Five (somewhat) arbitrary points $(n)$ were selected between 0 and 1 (i.e. $0 ; 0.19 ; 0.5 ; 0.77$ and 1) to define the shape of $g$ function by a cubic spline interpolation. The $g(n, f)$ function was calculated by adjusting $g(n, f)$ to experimental reflectance data, and the values of fitting are also indicated in the table.

Table S2: Simulated parameters of a-C:MeC with Bergman representation including the percolation strength $\left(g_{0}\right)$.

\begin{tabular}{lccccccc}
\hline \multicolumn{1}{c}{ Sample } & $\boldsymbol{g}_{\boldsymbol{0}}$ & $\boldsymbol{g}(\mathbf{0})$ & $\boldsymbol{g ( \mathbf { 0 . 1 9 } )}$ & $\boldsymbol{g ( \mathbf { 0 . 5 } )}$ & $\boldsymbol{g ( \mathbf { 0 . 7 7 } )}$ & $\boldsymbol{g ( \mathbf { 1 } )}$ & $\begin{array}{c}\text { Simulated / measured } \\
\text { carbide VF (\%) }\end{array}$ \\
\hline a-C:VC (21 at.\%V) & 1 & 0.34 & 0.99 & 0.67 & 0.57 & 0.83 & $43 / 40$ \\
a-C:VC (6 at.\%V) & 0 & 0.83 & 0.93 & 0.85 & 1.00 & 0.93 & $14 / 12$ \\
C:Mo (19 at.\%Mo) & 1 & 0.19 & 0.99 & 0.00 & 0.78 & 0.51 & $43 / 40$ \\
C:Mo (3 at.\%) & 0 & 0.00 & 1.00 & 0.99 & 0.00 & 0.99 & $10 / 7$ \\
\hline
\end{tabular}

\section{References}

[1] G. Katumba, A. Forbes, Carbon-in-silica composite selective solar absorbers: a determination of composition and dielectric properties, in: B.K. Tsai (Ed.), Soc. Photo-Optical Instrum. Eng., 2009: p. 74100G-74100G-9.

[2] J.C. Maxwell-Garnett, Colours in Metal Glasses, in Metallic Films, and in Metallic Solutions. II, Philos. Trans. R. Soc. A Math. Phys. Eng. Sci. 205 (1906) 237-288.

[3] D. Bruggeman, Calculation of various physics constants in heterogenous substances I Dielectricity constants and conductivity of mixed bodies from isotropic substances, Ann. Phys. 24 (1935) 636-664.

[4] I. Heras, E. Guillén, G. Abrasonis, M. Krause, A. Pardo, J.L. Endrino, et al., Optimizing solar absorption in carbon transition metal nanocomposite thin films, Sol. Energy Mater. Sol. Cells. (2016).

[5] D. Bergman, Dielectric - Constant if a Composite material - Problem in classical Physicis, Phys. Reports Rev. Sect. Phys. Lett. 43 (1978) 378-407.

[6] J. Sturm, P. Grosse, W. Theiss, Effective dielectric functions of alkali halide composites and their spectral representation, Zeitschrift Für Phys. B Condens. Matter. 83 (1991) 361-365.

[7] W. Theiss, S. Henkel, M. Arntzen, Connecting microscopic and macroscopic properties of porous media: choosing appropriate effective medium concepts, Thin Solid Films. 255 (1995) 177-180.

[8] G. a Niklasson, C.G. Granqvist, Optical properties and solar selectivity of coevaporated Co-Al2O3 composite films, J. Appl. Phys. 55 (1984) 3382-3410. 


\section{Advanced characterization and optical simulation for the design of solar selective coatings based on carbon: transition metal carbide nanocomposites}

Heras, I.

Elsevier

I. Heras, M. Krause, G. Abrasonis, A. Pardo, J.L. Endrino, E. Guillén, R. Escobar-Galindo, Advanced characterization and optical simulation for the design of solar selective coatings based on carbon: transition metal carbide nanocomposites, Solar Energy Materials and Solar Cells, Volume 157, December 2016, Pages 580-590 http://dx.doi.org/10.1016/j.solmat.2016.07.011.

Downloaded from Cranfield Library Services E-Repository 